\title{
Kollaboratives Schreiben
}

\author{
Claas Morgenroth
}

I.

Man schreibt nie allein. Eine eigene Textsorte, die Danksagung, bringt diesen Umstand zum Ausdruck. Heinrich Bosse zum Beispiel leitet seine Aufsatzsammlung Bildungsrevolution 1770-1830 mit den Worten ein: „Im Grunde wissen alle, daß man als Autor von solchen Dingen [das sind hier Bücher; C. M.] ebenfalls nicht einer, sondern ein Kollektivsubjekt ist. Dieser Moment, wo ich mit ,meinen' Texten an die Öffentlichkeit trete, ist der gegebene Augenblick, um anzuerkennen, wie wenig sie ,meine‘ sind.." Die Gründe liegen auf der Hand: Ein Autor gehört immer zu einer Tradition, einer Epoche, einer Zeit; er bezieht sich stets, negativ wie positiv, auf Vorbilder, andere Texte und Autoren; er hat, je nach Disposition, ein Publikum vor Augen, für das und in dessen Vorstellung er schreibt; er wird schließlich mit Akteuren konfrontiert - Verleger, Lektor, Kritiker -, die mal mehr, mal weniger seine Arbeit beeinflussen. Schreiben, so könnte man sagen, ist daher immer kollaborativ, kooperativ, kollektiv.

Die These ist beileibe nicht neu, im Gegenteil: Man findet sie in der Autorschaftsdebatte, der Intertextualitätstheorie, in allen Text-Kontext-Modellen, ja eigentlich in jeder Literaturtheorie. Und sie reicht weit zurück in die Geschichte der Poetik. Darunter sind prominente Beispiele, die zeigen, dass allerorten Götter, Geister oder zumindest Regeln auftreten, die dem angehenden Autor erlauben und diktieren, was er schreiben soll und darf. Martin Opitz' Buch von der Deutschen Poeterey, die erste ernstzunehmende Poetik in deutscher Sprache, ist nicht nur eine Regelkunde, sondern selbst ein Referat überlieferter Poetiken - was Opitz im Übrigen gar nicht leugnet. Sie geht so weit, ein Kapitel aus Julius Caesar Scaligers Sieben Bücher über die Dichtkunst ${ }^{2}$ abzuschreiben, das sich wiederum als Summe antiker Poetik und Rhetorik versteht.

1 Heinrich Bosse, Bildungsrevolution 1770-1830, hrsg. von Nacim Ghanbari, Heidelberg: Winter 2012, S. VII-IX, hier S. VII.

2 Julius Caesar Scaliger, Poetices libri septem. Sieben Bücher über die Dichtkunst (1561), Lateinisch/Deutsch, hrsg., übers., eingel. und erläutert von Luc Deitz, 6 Bde., Stuttgart-Bad Cannstatt: Frommann-Holzboog 1994-2011; die hervorragende, von Luc Deitz in Zusammenarbeit mit Gregor Vogt-Spira verfasste „Einführung“, Bd. 1, S. XI-LXXIV, bietet nebenbei gesagt einen ausgezeichneten Einblick in die europäische Poetik und Abschreibepraxis des ausgehenden 16. und 17. Jahrhunderts. 
„Derentwegen ich nur etwas / so ich in gemeine von aller Poeterey zue erinnern von noethen zue sein erachte / hiervor setzen will / nachmals das was vnsere deutsche Sprache vornemlich angehet / etwas vmstendtlicher fuer Augen stellen.“3 Erst mit der Querelle des Anciens et des Modernes wird der Imitationszwang der Antike fundamental erschüttert, mit weitreichenden Folgen für die Ästhetik des 18. Jahrhunderts. ${ }^{4}$ Aber noch am Ausgang der traditionellen Regelpoetik, der vierten Auflage von Johann Christoph Gottscheds Critischer Dichtkunst, findet sich zuerst eine Übersetzung von Horaz' Ars Poetica, zu der sich Gottscheds Traktat im Weiteren wie ein Kommentar verhält. ${ }^{5}$

Ko-Autorschaft in diesem allgemeinen Sinne ist alles andere als eine Entdeckung der Moderne. Sie führt zurück zu den Anfängen der Literatur, zur „Kollektiven Autorschaft im Alten Testament" oder zu den Anfangsworten der Odyssee, „Muse! Erzähl' mir vom wendigen Mann, der die heilige Feste / Trojas zerstörte!"6 Das vielleicht schönste Beispiel bieten die zwei Niederschriften der Zehn Gebote, die sehr genau unterscheiden zwischen einem Autor und einem Schreiber: „Und als der Herr mit Mose zu Ende geredet hatte auf dem Berge Sinai, gab er ihm die beiden Tafeln des Gesetzes; die waren aus Stein und beschrieben von dem Finger Gottes." Die Zeit der Niederschrift aber dauert lange, zu lange. „Mose wandte sich und stieg vom Berge und hatte die zwei Tafeln des Gesetzes in seiner Hand; die waren beschrieben auf beiden Seiten.

3 Martin Opitz, Buch von der Deutschen Poeterey (1624), Studienausgabe, hrsg. von Herbert Jaumann, Stuttgart: Reclam 2002, S. 13.

4 Zur Bedeutung der Querelle Hans Ulrich Gumbrecht, „Modern, Modernität, Moderne“, in: Otto Brunner, Werner Conze und Reinhart Koselleck (Hrsg.), Geschichtliche Grundbegriffe. Historisches Lexikon zur politisch-sozialen Sprache in Deutschland, Bd. IV, Stuttgart: KlettCotta 1978, S. 93-131.

5 Johann Christoph Gottsched, Versuch einer Critischen Dichtkunst, unveränderter photomechanischer Nachdruck der 4., vermehrten Auflage, Leipzig 1751, Darmstadt: Wissenschaftliche Buchgesellschaft 1962. Man denke bei dieser Gelegenheit an Walter Benjamin: "Geschichte schreiben heißt also Geschichte zitieren. Im Begriff des Zitierens liegt aber, daß der jeweilige historische Gegenstand aus seinem Zusammenhange gerissen wird" (Walter Benjamin, Das Passagen-Werk (1927-1940), hrsg. von Rolf Tiedemann, Frankfurt/Main: Suhrkamp 1983, S. 595, N 11, 3; Herv. im Orig.).

6 Ulrich Berges, „Kollektive Autorschaft im Alten Testament“, in: Christel Meier und Martina Wagner-Egelhaaf (Hrsg.), Autorschaft. Ikonen - Stile - Institutionen, Berlin: Akademie 2011, S. 29-39; Homer, Odyssee und Homerische Hymnen, aus dem Altgriechischen übersetzt von Anton Weiher, München: dtv 199o, S. 51. Mit diesen Worten Homers (wer auch immer ,Homer' ist) tritt zugleich die Unterscheidung von Autor und Schriftsteller in die Welt, siehe dazu: Martin Stingelin, „,er war im Grunde der eigentliche Schriftsteller, während ich bloss der Autor war. Friedrich Nietzsches Poetologie der Autorschaft als Paradigma des französischen Poststrukturalismus (Roland Barthes, Gilles Deleuze, Michel Foucault)“, in: Heinrich Detering (Hrsg.), Autorschaft: Positionen und Revisionen, DFG-Symposion 2001, Stuttgart, Weimar: Metzler 2002, S. 80-106. 
Und Gott hatte sie selbst gemacht und selber die Schrift eingegraben. [...] Als Mose aber nahe zum Lager [des Volkes Israel; C. M.] kam und das Kalb und das Tanzen sah, entbrannte sein Zorn und er warf die Tafeln aus der Hand und zerbrach sie unten am Berge“. So kommt es zu einer zweiten Fassung der Zehn Gebote, die nun von Gott diktiert und von Mose aufgeschrieben werden. „Und der Herr sprach zu Mose: Schreib dir diese Worte auf, denn aufgrund dieser Worte habe ich mit Dir und mit Israel einen Bund geschlossen. [...] Und er schrieb auf die Tafeln die Worte des Bundes, die zehn Gebote.“7

Warum also befasst man sich heute wieder so intensiv mit den verschiedenen Formen gemeinsamen Schreibens, warum geht man davon aus, es mit einer Novität und einem Desiderat der Forschung zu tun zu haben? Es gibt dafür verschiedene Gründe, zwei aber stechen heraus: Seit der ,Bildungsrevolution 1770-1830' gehört das Schreiben zum individualisierten, autonomen Subjekt. So prominent die Beispiele zur Ko-Autorschaft auch sein mögen, von der Antike bis zur Frühaufklärung, seit der Wende zum 19. Jahrhundert gilt das gemeinsame Schreiben als Sonderfall, selbst in der kommunistischen und marxistisch inspirierten Ästhetik. ${ }^{8}$ Wenn man sich heute wieder den

7 Die Bibel, nach der Übersetzung Martin Luthers, mit Apokryphen, in der revidierten Fassung von 1984, hrsg. von der Evangelischen Kirche in Deutschland, Stuttgart: Deutsche Bibelgesellschaft 1999, S. 91-95, 2. Mose 31, 18; 32, 15f. und 19; 34, 27f. Die Szene wiederholt sich, halbwegs zumindest, unter reichlich komischen Umständen in Vassula Rydens True Life in God (hier ist es Jesus, der diktiert, und Ryden, die in zwei Handschriften schreibt, ihrer und der von Jesus). Dazu Andreas Mauz, „Federführung, Grenzüberschreitung. Schreiben und Schreibenanfangen im ,heiligen Text" (Vassula Ryden, True Life in God, 1986ff.)“, in: Hubert Thüring, Corinna Jäger-Trees und Michael Schläfli (Hrsg.), Anfangen zu schreiben. Ein kardinales Moment von Textgenese und Schreibprozeß im literarischen Archiv des 20.Jahrhunderts, München: Fink 2009, S. 43-76.

8 Vgl. dazu Bernd Witte, Goethe. Das Individuum der Moderne schreiben, hrsg. von Verf. und Karl Solibakke, Würzburg: Königshausen \& Neumann 2007; Richard Albrecht, „Vom Roman der XII zum Kollektivroman Wir lassen uns nicht verschaukeln: Aspekte literarischer Gemeinschaftsproduktionen in der deutschen Literatur des 20. Jahrhunderts“, in: Neohelicon 14 (1987), H. 1, S. 269-285. Auch die Auseinandersetzungen zum ExpressionismusBegriff und zum sozialistischen Realismus sind Beispiele dafür, wie umkämpft das Verhältnis des individualisierten Schreibers zum Kollektiv, zur Gesellschaft, zur Allgemeinheit in der marxistischen Literaturtheorie war. Siehe dazu nur Walter Hinderer (Hrsg.), SickingenDebatte. Ein Beitrag zur materialistischen Literaturtheorie, Darmstadt, Neuwied: Luchterhand 1974, sowie Mary A. Nicholas und Cynthia A. Ruder, „In Search of the Collective Author: Fact and Fiction from the Soviet 1930s“, in: Book History 11 (2008), S. 221-244. Zembylas und Dürr, die zu den wenigen gehören, die sich empirisch mit der Praxis des literarischen Schreibens beschäftigt haben, konzentrieren sich allein auf den individualisierten Autor. Kollaborative Elemente werden nicht berücksichtigt: Tasos Zembylas und Claudia Dürr, Wissen, Können und literarisches Schreiben. Eine Epistemologie der künstlerischen Praxis, Wien: Passagen 2009 . 
verschiedenen Formen des geteilten Schreibens zuwendet, dann um dieser Geschichte der Autonomie kritisch zu begegnen. ${ }^{9}$ Ein Ansatz, der sich gut mit den subjektskeptischen Theorieansätzen der letzten $5^{\circ}$ Jahre verträgt (und damit wissenschaftsstrategischen Erfolg verspricht). Der zweite Grund ist in der Konstitution heutiger Gesellschaften zu suchen (natürlich nicht aller), die zunehmend Wert auf kooperative und kollaborative Arbeitsformen legen, angeleitet oder in Gang gesetzt durch computerisierte, schriftbasierte Praktiken und Kulturtechniken, in Unternehmen und Schulen etwa. Eins und zwei verbinden sich mit einem Diskurs, der auf Pluralität, Vielheit, Diversität gestimmt ist. So fragt sich Mark Terkessidis in seinem Buch Kollaboration: „Sind alle unsere Institutionen, sind Behörden, Schulen oder Gesundheitseinrichtungen ,fit für die Vielheit? Und wenn nicht: Was müssen sie unternehmen, um es zu werden?"10

Das Spektrum ist damit nicht erschöpft, aber das Panorama erkennbar und mit einem ironischen Vorzeichen versehen. Schließlich steht die Fitness des Einzelnen und der Institutionen im Kreuzungspunkt eines postfordistischen, gouvernementalen Diskurses, der die Selbstoptimierung des Subjekts zur Bedingung und Folge der Kollektivierung im kapitalistischen Gesellschaftskörper macht..11 Mit dem Umbau der Arbeitswelt wird das Individuum liberalisiert und in Verantwortung gesetzt und zugleich das individualistische Selbstverständnis der Moderne in Frage gestellt, in der Poetik wie in der ökonomischen Ordnung. An dieser Dialektik werden sich jene Fragen messen lassen müssen, die mit dem geteilten Schreiben als Arbeit befasst sind. Und was soll Schreiben sein, wenn nicht Arbeit?

$9 \quad$ Zuweilen etwas voreilig, schließlich kann der Gewinn an Einsamkeit im Schreiben auch einen Gewinn an Individualität und Emanzipation bedeuten. Siehe dazu nur Virginia Woolf, A Room of One's Own (1926), deutsch: Ein Zimmer für sich allein, aus dem Englischen übersetzt, mit einem Nachwort und Anmerkungen von Axel Monte, Stuttgart: Reclam 2012, und im Vergleich Bettine von Arnim, Goethe's Briefwechsel mit einem Kinde (1835), in: dies., Werke und Briefe, hrsg. von Walter Schmitz, Bd. 2, Frankfurt/Main: Deutscher Klassiker Verlag 1992. Dabei ist der Begriff ,Autonomie‘ selbst kritikwürdig, z.B. als Effekt „bürgerlicher Technologien des Selbst“ (Andreas Reckwitz, Das hybride Subjekt. Eine Theorie der Subjektkulturen von der bürgerlichen Moderne zur Postmoderne, Weilerswist: Velbrück Wissenschaft 2006, S. 155-241).

10 Mark Terkessidis, Kollaboration, Berlin: Suhrkamp 2015, S. 10.

11 Zum Begriff der Gouvernementalität Michel Foucault, „Über sich selbst schreiben“ (Lécriture de soi, 1983), aus dem Französischen übersetzt von Michael Bischoff, in: ders., Schriften in vier Bänden. Dits et Ecrits, Bd. IV, hrsg. von Daniel Defert und François Ewald, unter Mitarbeit von Jacques Lagrange, Frankfurt/Main: Suhrkamp 2005, S. 503-521, in Verbindung zur kapitalistischen Ökonomie: Thomas Lemke, Eine Kritik der politischen Vernunft. Foucaults Analyse der modernen Gouvernementalität, Berlin, Hamburg: Argument 1997 . 
In den Literatur- und Kulturwissenschaften kursiert nun eine Reihe von Begriffen und Modellen, die versuchen, das Schreiben als eine irgendwie geteilte, zumindest nicht mehr individuelle Praxis zu begreifen. Es gehört dabei zum guten Ton, die Subjektivität und Vereinzelung des Schreibens als Chimäre einer bis dato übersehenen Linie über- oder transindividueller Techniken und Praktiken zu begreifen. ${ }^{12}$ Das Schreiben dient dabei, wie gewohnt, als besonderer Ausdruck einer ins Allgemeine gehenden Tendenz in der Theoriebildung. Ziel ist, die Versprechen des Idealismus auf eine autonome, an der Freiheit des Geistes oder der Emanzipation bemessene Existenz wieder einzuholen. Allerdings nicht aus einer materialistischen Perspektive (Stichwort: Eigentumsverhältnisse), sondern aus einer ,materialen', also unter Verzicht auf historisch-materialistisch und/oder dialektisch orientierte Methoden..$^{13}$ Das führt zur Marginalisierung von ,Arbeit'. Dazu zwei Schlaglichter:

Der Begriff ,Kulturtechniken' verkehrt die Subjekt-Objekt-Relation (oder auch: Mensch-Ding-Relation), um die je spezifische Subjektivität des Einzelnen in einem kollektiven Handlungsraum aufzulösen, der aus einer Vielzahl an (belebten wie unbelebten) Akteuren besteht.

Kulturtechniken präzisieren die Handlungsmacht von Medien und Dingen. Wäre oder hätte die Medientheorie eine Grammatik, so käme diese Handlungsmacht darin zum Ausdruck, dass Objekte die grammatikalische Stellung des Subjekts einnehmen und Kulturtechniken Verben vertreten. Personen (gleich Menschen) rücken darin an jene Stelle in einem Satz, die für das grammatikalische Objekt reserviert ist. ${ }^{14}$

Das Schreiben ist so gesehen nicht mehr am Einzelnen zu beobachten, sondern als Prozess oder Bewegung zu begreifen, an der eine ganze Reihe an Subjekten

12 Dafür stehen insbesondere die Arbeiten von Bruno Latour und Harold Garfinkel. Siehe dazu u.a. Navigationen 15 (2015), H. 1, Medien der Kooperation.

13 Zur Aktualität der Unterscheidung materialistisch/material und zur Diskussionslage des material turn aus marxistischer Perspektive Terry Eagleton, Materialism, New Haven, London: Yale University Press 2016, insbes. S. 1-35, und Martin Küpper, Materialismus, Köln: PapyRossa 2017, S. 112-121.

14 Cornelia Vismann, „Kulturtechniken und Souveränität“, in: dies., Das Recht und seine Mittel, hrsg. von Markus Krajewski und Fabian Steinhauer, Frankfurt/Main: S. Fischer 2012, S. 445-459, hier S. 445; zuerst in: Zeitschrift für Medien- und Kulturforschung 1 (2010), S. 171-182. Und weiter: „Diese Vertauschung der Personen ist vielleicht das offensichtlichste Kennzeichen einer Theorie der Kulturtechniken, von den Medien aus betrachtet." Siehe auch Bernhard Siegert, „Kulturtechnik“, in: Harun Maye und Leander Scholz (Hrsg.), Einführung in die Kulturwissenschaft, München: Fink 2011, S. 95-118, hier S. 98: „Medien werden als Kulturtechniken beschreibbar, wenn die Handlungsketten rekonstruiert werden, in die sie eingebunden sind, die sie konfigurieren oder die sie konstitutiv hervorbringen." 
und Objekten in unterschiedlichen Relationen beteiligt sind, ein Schreiber etwa, Stifte, Papiere, Tische und Stühle. ${ }^{15}$,Netzwerk' wird dagegen bemüht, um vor- oder semi-institutionelle Formen des Schreibens und der ,Literatur zu erfassen. Die literarische Aufklärung und Romantik stehen hier zur Stelle, um die überzogene Individualisierung um 1800 und die auf den Autor und das Werk hierarchisierte Wahrnehmung des Schreibens im Büchermarkt des 19. und 2o. Jahrhunderts, zumindest im deutschsprachigen Raum, zu revidieren. In der Sache handelt es sich um die nächste Kritik am Idealismus, der von der Macht der Medien, Dinge, Techniken, Hardware ablenkt.. ${ }^{16}$ Das ist nützlich, weil auf diese Weise vergessene und übersehene Praktiken der Literatur und Kunst wieder sichtbar werden. Verzichtet wird dabei auf eine ,gesellschaftliche', oder sagen wir besser, mit einem reichlich angestaubten Wort: sozioökonomische Analyse der jeweiligen Arbeits- und Eigentumsverhältnisse, die den Praktiken des Schreibens zu Grunde liegen oder zumindest beiwohnen.

Der Anlass solcher turns, ihr ökonomischer Impuls, wird nicht ausreichend oder gar nicht berücksichtigt - eine alte Schwäche der kultur- und literaturwissenschaftlichen Begriffsbildung. An der Vorder- und Rückseite des Netzwerkbegriffs ist das zu beobachten: Gebildet, um bürgerlichen Ordnungsbegriffen und Strukturen eine Alternative abzuringen, wird er fortgeführt und neoliberal adaptiert, um den Nutzen am menschlichen Material zu optimieren. ${ }^{17}$ Das mag an dieser Stelle zu pointiert formuliert sein und der Vielseitigkeit des Netzwerkbegriffs nicht gerecht werden, aber es fällt auf, dass in der Welt des akademischen Kapitalismus die ins Spiel gebrachten Ideen und Konzepte, Begriffe, Innovationen und Desiderate ausgesprochen dürftig auf ihr ökonomisches Herkunftsfeld geprüft werden. Die Literatur- und Kulturwissenschaften tun gut daran, ihre Begriffe (wieder) an der ökonomischen und politischen Wirklichkeit zu messen. ${ }^{18}$

15 Dazu in aller Ausführlichkeit Roland Barthes, Die Vorbereitung des Romans. Vorlesung am Collège de France 1978-1979 und 1979-1980, hrsg. von Éric Marty, Texterstellung, Anmerkungen und Vorwort von Nathalie Léger, aus dem Französischen übersetzt von Horst Brühmann, Frankfurt/Main: Suhrkamp 2008.

16 Michael Gamper, „Kollektive Autorschaft/Kollektive Intelligenz: 180o-200o“, in:Jahrbuch der Deutschen Schillergesellschaft 45 (2001), S. 380-403; Nacim Ghanbari et al. (Hrsg.), Kollaboration. Beiträge zur Medientheorie und Kulturgeschichte der Zusammenarbeit, Paderborn: Fink 2018; Zeitschrift für Germanistik, N.F. 29 (2019), H. 1, Schwerpunkt: Werke in Relationen. Netzwerktheoretische Ansätze in der Literaturwissenschaft.

17 Dazu Stefan Kaufmann, „Netzwerk“, in: Ulrich Bröckling, Susanne Krasmann und Thomas Lemke (Hrsg.), Glossar der Gegenwart, Frankfurt/Main: Suhrkamp 2004, S. 182-189.

18 Zur Erinnerung: Richard Münch, Akademischer Kapitalismus. Über die politische Ökonomie der Hochschulreform, Frankfurt/Main: Suhrkamp 2o11, und Mathias Binswanger, Sinnlose Wettbewerbe. Warum wir immer mehr Unsinn produzieren, Freiburg: Herder 2010. 


\section{II.}

Viel hängt davon ab, ein Gemeinplatz der Schreibprozessforschung, wie metaphorisch oder wie konkret der Begriff des Schreibens verstanden wird, wie historisch informiert er ist. Angesichts der aufgebotenen "Theorielast" dürfte es klüger sein, sich auf den konkreten Schreibakt zu konzentrieren, um von dort aus die kulturtechnischen und, vernetzten' Aspekte des Schreibens aufzugreifen. Entsprechend sinnvoll ist es, voraussetzungsstarke Ausdrücke wie ,Autorschaft', ,geteiltes' oder ,gemeinsames Schreiben' nicht weiter zu vermengen. Ausgangspunkt der weiteren Überlegungen ist darum ein Vorschlag, die zunächst einmal rudimentäre Unterscheidung von ,kollaborativem', ,kooperativem' und ,kollektivem' Schreiben. Mit ,kollaborativ' sei die über den Einzelnen hinausgehende ( $k o-)$ Arbeit gemeint, die entsprechend angebahnt, organisiert, konzipiert und durchgeführt werden muss, auf allen Ebenen des Schreibprozesses und im Verhältnis zur gesellschaftlich dominanten Arbeitsweise. Der Begriff der ,Kooperation“ bezeichne dagegen die im Werk/ Produkt (opus) vergegenständlichte, semantisierte, erzählte, dokumentierte Kollaboration. Zu bedenken ist, dass im kollaborativen Schreiben die Gestalt und der Status des Werkes/Produktes - mit der Linguistik gesprochen: des Textes - eine tragende Rolle spielen kann, wenn etwa die Kollaboration bereits auf Kooperation angelegt ist. Das kann die zeitliche Organisation des Schreibens (synchron wie asynchron), deren räumliche Gestaltung (Vernetzung und Koordination) oder Medialisierung betreffen. ,Kollektiv' sollen Schreibprozesse dann heißen, wenn die Kollaboration und Kooperation zu Gunsten einer Gruppe (von der Belegschaft bis zum ,Volk'), eines übergeordneten Projekts, einer höheren Idee usf. ausgeführt wird - ein Umstand, der sich wiederum auf die einzelnen Schritte des Schreibprozesses auswirken kann. ${ }^{19}$

Die Unterscheidung mag auf den ersten Blick idiosynkratisch sein. Ihr Ziel aber ist, die vielfachen Assoziationsangebote aus anderen Theoriefeldern (Intertextualität, Autorschaft) zunächst einmal außen vor zu lassen, um den Akt, die Szene, das Ereignis des Schreibens genauer in den Blick zu nehmen und zu beschreiben. Hilfe bietet die literaturwissenschaftliche

19 Diese Unterscheidung ist das Ergebnis einer Dortmunder Arbeitsgruppe gewesen, die sich in unterschiedlichen Besetzungen (aber jeweils unter der Teilnahme von Tobias Lachmann und Martin Stingelin) mit dem Thema befasst hat. Den genannten und ungenannten Kolleginnen und Kollegen gilt mein Dank für die zahlreichen Diskussionen. Was hier nicht weiter erörtert werden kann, zu kollektiven Schreibprozessen aber gezählt werden muss, ist deren Vergegenständlichung in Kollektivsymbolen, dazu Jürgen Link, „Kollektivsymbol und Mediendiskurs“, in: kultuRRevolution 1 (1982), S. 6-21. 
Schreibprozessforschung (dazu später) und die linguistische Theoriebildung, die sich bereits seit den 1970er Jahren intensiv und umfassend mit Aspekten gemeinsamen Schreibens befasst - und in diesem Punkt den Literatur- und Kulturwissenschaften voraus ist. ${ }^{20}$

In einem Beitrag für das Handbook of Writing and Text Production schlagen Kirsten Schindler und Joanna Wolfe vor, die verschiedenen Dimensionen geteilten Schreibens am Beispiel der Ko-Autorschaft/Ko-Verfasserschaft (oder auch: „co-authored, or collaborative writing“) zu untersuchen und zu systematisieren. Sie räumen zugleich ein, dass es schwierig ist, den Begriff zu definieren, weil ,Schreiben' im Grunde immer auf einer gemeinsamen ,Verfasserschaft' beruhe, man sich stets in bestimmten Genres, Gattungen oder Textsorten bewege, Teil eines Diskurses, immer schon der Leser anderer Texte ist usw. Insofern sei es notwendig, die Fragestellung einzugrenzen und weiter zu spezifizieren. Demnach sind ,ko-autorisierte‘ oder ,kollaborative‘ Texte im Weiteren nur noch ,kollaborativ', um den Assoziationen zur Autorschaftsdebatte in den Literaturwissenschaften zu entgehen - solche Dokumente, in denen mehrere Individuen im Vorgang des Schreibens direkt interagieren, beim Planen, Entwerfen oder Überarbeiten. Konkret: wenn zwei oder

20 Interessanterweise handelt es sich bei den linguistischen Beiträgen vor allem um solche, die mit dem Schreibunterricht an Schulen befasst sind, oder allgemeiner: mit der Didaktik und den Methoden des Schreibens. So widmet sich die Zeitschrift Written Communication schon lange und ausgiebig Themen wie Schreibkonferenz, Chat-Kommunikation, OnlineKonferenzen, Digitale Schreibräume an Schulen, Videoaufzeichnung und -dokumentation von (gemeinsamem) Schreiben/Lesen im Klassenraum. Hier bewährt sich, was auch sonst zu beobachten ist: Die schreibdidaktischen Orientierungsbegriffe waren und sind über die eigenen disziplinären Grenzen hinaus ein nützlicher Indikator für die allgemeine Transformation des Schreibens in Theorie und Methode. Nur drei Hinweise: Angela M. O'Donnell et al., „Cooperative Writing. Direct Effects and Transfer“, in: Written Communication 2/3 (1985), S. 307-315; Colette Daiute, „Do 1 and 1 Make 2? Patterns of Influence by Collaborative Authors“, in: Written Communication 3/3 (1986), S. 382-408; schließlich Peter Elbow, „In Defense of Private Writing. Consequences for Theory and Research“, in: Written Communication 16/2 (1999), S. 139-170. Die linguistische Schreibdidaktik unterscheidet sich damit von der Texttheorie, die sich weiter an traditionelle Einsamkeitsmodelle gebunden sieht, gerade dann, wenn es um, Literatur' geht. Bei Hans Jürgen Heringer ist zu lesen: „Texte werden immer von Einzelpersonen geschrieben“ (Hans Jürgen Heringer, Linguistische Texttheorie. Eine Einführung, Tübingen: Francke 2015, S. 204). Auch Manfred Geier ist in seiner, wenngleich deutlich älteren, Einführung der Auffassung: Die „Texte der Literatur [sind], zumindest in der Moderne, bloß partikular, gleichsam stilistische Ideolekte von Einzelnen, die nicht zufällig die literarische Praxis mit ihrem Namen autorisieren“ (Manfred Geier, Linguistische Analyse und literarische Praxis. Eine Orientierungsgrundlage für das Studium von Sprache und Literatur, Tübingen: Narr 1984, S. 8). In der schmalen Literatur zur Soziologie des Schreibens ist man der derselben Auffassung, siehe nur Zembylas/Dürr, Wissen, Können (Anm. 8). 
mehrere Personen an einem Dokument arbeiten, wenn einer oder mehrere ein von jemand anderem erstelltes Dokument bearbeiten und ändern, einer mit einem oder mehreren interaktiv zusammenarbeitet und dabei ein Dokument erstellt, das auf die Ideen eines anderen oder anderer zurückgeht. ${ }^{21}$ Kollaboratives Schreiben kann gemeinsam und simultan erfolgen; parallel und unabhängig voneinander, etwa dann, wenn jeder der Beteiligten für einen Teil des Dokuments verantwortlich ist; zentralisiert, wenn es letztlich nur einen verantwortlichen Autor gibt; gestaffelt, wenn Dokumente von Person zu Person wandern zwecks Überarbeitung und Fortsetzung. ${ }^{22}$ Ihre Beispiele suchen sich Wolfe und Schindler in den Wissenschaften (Artikel in der Medizin, Forschungsanträge), in Unternehmen (Rechenschaftsbericht, Selbstdarstellungen), in der Werbung - in Bereichen also, in denen kollaboratives Schreiben eher die Regel als die Ausnahme ist.

Aus diversen Studien ergebe sich dabei, dass die Wahl der jeweiligen Strategie abhängt von der Textsorte, vom Ziel, der Zusammensetzung der Gruppe (hierarchisch oder nicht), oder auch wechseln kann, je nachdem, in welchem Stadium des Schreibens man sich befindet (planen, ausarbeiten, redigieren). Besondere Aufmerksamkeit erhält das Planen, schließlich benötigen kollaborative Arbeitsformen gesteigerte Vorbereitung und Organisation. Dabei entstehen im Zuge der Anbahnung und Durchführung wiederum Texte, die dafür da sind, die Zusammenarbeit zu ermöglichen oder zu unterstützen (z.B. Protokolle, Memos, Briefings, allgemein: Arbeitspläne). ${ }^{23}$ So müssen sich im Fortgang kollaborativen Schreibens die Teilnehmerinnen

21 Kirsten Schindler und Joanna Wolfe, „Beyond Single Authors: Organizational Text Production as Collaborative Writing“, in: Eva-Maria Jakobs und Daniel Perrin (Hrsg.), Handbook of Writing and Text Production, Berlin, Boston: de Gruyter 2014, S. 159-173. Das Zitat "Co-authored, or collaborative writing" steht auf S. 160.

22 „Four basic collaborative strategies co-authors use to coordinate their efforts: - Joint: Several people simultaneously work on the entire document, thus sharing joint control. This strategy is also referred to as collective co-authoring [...]. - Parallel: Several people independently work on individual sections of a document, also referred to as horizontaldivision [...]. - Centralized: One person maintains responsibility for writing the document; others may offer suggestions or criticism, but these are always directed to the primary author. - Relay: One person works on the document and then passes it along to another team member for revisions and additions, and so on. This strategy has also been referred to as sequential working $[\ldots]$, serial collaboration [...], and document cycling" (Schindler/Wolfe, „Beyond Single Authors“ [Anm. 21], S. 161f.).

23 Dazu aus Sicht der Literaturwissenschaft Rolf Parr, „Das Protokoll als literarische Kunstform. Zur Konvergenz von künstlerischer und juridischer Selbstvergewisserung in literarisch-kulturellen Vereinen des 19. Jahrhunderts“, in: Michael Niehaus und HansWalter Schmidt-Hannisa (Hrsg.), Das Protokoll. Kulturelle Funktionen einer Textsorte, Frankfurt/Main u.a.: Lang 2005, S. 167-186, und Gundel Mattenklott, „Im Labyrinth der 
immer wieder treffen oder anderweitig verbinden, um sich mündlich oder schriftlich über bereits Geschriebenes zu verständigen. Hier ist es besonders wichtig, das Gesagte wieder aufzuschreiben, um in der Schrift Verbindlichkeit herzustellen; dergleichen beim Redigieren und Überarbeiten. Alles in allem erweise sich, so Schindler und Wolfe, die Komplexität kollaborativen Schreibens an den kleinen Schritten der Textproduktion, deren Modellierung wiederum didaktischen Wert habe.

Der didaktisierende Anspruch hat historische Gründe, die von Wolfe und Schindler nicht weiter thematisiert werden, für eine poetisch-linguistische oder auch literaturwissenschaftliche Analyse aber von Bedeutung sind. ${ }^{24}$ In den USA machte man sich Mitte der 197oer Jahre Sorgen um die Schreibkompetenz angehender Schüler. „Why Johnny can't write“, titelte Newsweek und provozierte damit erstaunliche (auch monetäre) Anstrengungen im Bildungssystem. ${ }^{25}$ Dem Problem stand bald eine empirisch ausgelegte, zugleich konstruktivistische Forschungslandschaft gegenüber, die gut zur neoliberalen Politik der ReaganÄra passte. Von der Durchleuchtung des Schreibens versprach man sich eine Optimierung von Unterricht und Leistung, einschließlich Erprobung, Prüfung und Evaluation, kurz: Management. ${ }^{26}$ Hinter der Modellierung verschwand sowohl die Geschichte des Schreibens als auch die Geschichte des Schreibens als Lohnarbeit. Dabei ist diese Unterteilung - planen, ausarbeiten, redigieren von ihren Anfängen her Gegenstand der Rhetorik und hat eine Entwicklung durchlaufen, die für das Verständnis von Arbeit und Schreiben unverzichtbar ist. Otto Ludwig weist in seiner kurzen "Geschichte des Schreibens" darauf hin. „Was die Zeit nach 1150 auszeichnet, sind die Veränderungen im produktiven Bereich. Der Autor wird zum Schreiber. Er verwandelt sich vom ,Diktator` zu einer frühen Form des Schriftstellers." Und weiter:

Solange Schreiben arbeitsteilig vollzogen wurde, hatten die einzelnen Aktivitäten einen hohen Grad an Eigenständigkeit. Die Korrekturarbeiten hoben sich deutlich von den Schreibarbeiten im engeren Sinne des Wortes ab: der Nieder- und Reinschrift. Und diese wiederum waren deutlich von den mentalen Vorgängen im Kopf des Autors abgesetzt. Man hatte noch nicht einmal einen Begriff für den Schreibprozeß als ganzen und vermutlich auch nur wenig klare Vorstellungen von der Zusammengehörigkeit der einzelnen Vorgänge. Die

Begegnungen. Die Entstehung eines Gruppenromans“, in: Neue Sammlung 24/3 (1984), S. $262-28$ o.

24 Siehe dazu auch die Anmerkungen in Fußnote 18.

25 Merrill Sheils, „Why Johnny Can't Write“, in: Newsweek 92 (8.12.1975), S. 58-65.

26 Im Titel vereint bei Paul Benjamin Lowry, Aaron Curtis und Michelle René Lowry, „Building a Taxonomy and Nomenclature of Collaborative Writing to Improve Interdisciplinary Research and Practice“, in:Journal of Business Communication 41 (2004), H. 1, S. 66-99. 
Selbstständigkeit der verschiedenen Aktivitäten dürfte verhindert haben, daß die eine auf die andere einen nachhaltigen Einfluß nahm. Das mußte sich mit dem Augenblick ändern, in dem alle diese Aktivitäten von ein und derselben Person ausgeführt wurden. Nun war es möglich, daß sprachliche Korrekturen, selbst kompositorische oder gar konzeptionelle Veränderungen noch während der Niederschrift vorgenommen werden konnten [...]. Kurz: die Integration der verschiedenen Schreibarbeiten in einer einheitlichen, kontinuierlich sich entwickelnden Schreibhandlung führte zu einer Interaktion unter diesen und veränderte so den Schreibprozeß grundlegend. ${ }^{27}$

Diese Veränderung radikalisiert sich mit der Freisetzung des ,Autors‘ am Ende des 18. Jahrhunderts und setzt sich im ausgehenden 19. Jahrhundert an den Schulen durch, um dann wieder - zumindest in der Didaktik - in kooperativen Schreibformen aufgehoben zu werden. ${ }^{28}$ An dieser Stelle ist kein Platz für eine ausgiebige Diskussion dieser Geschichte. Nur so viel: Es ist bemerkenswert, dass und wie die Integration der verschiedenen Teilschritte des Schreibens in einer Hand sich parallel zur Industrialisierung und Teilung der Arbeitsprozesse entwickelt und im Zuge des Neoliberalismus wieder aufgespalten wird, obwohl oder weil die Autonomie des Einzelnen in der Ausbildung ökonomischer (und eben nicht mehr ästhetischer) Selbsttechniken aufgeht. So liegt es nahe, kollaboratives Schreiben im Wortsinn ernst zu nehmen, Schreiben also als Arbeit und Teil der Geschichte der Arbeit anzusehen. Schindler und Wolfe bieten den Schritt selbst an; sie sprechen von „Division of labor und ownership“, also Arbeitsteilung und Besitzverhältnisse, beschränken sich in der Auslegung ihrer Terminologie aber auf die Textproduktion (Textsorte, Adressierung des Produkts an einen/mehrere, Hierarchie und Ko-Autorschaft/ Ko-Verfasserschaft). ${ }^{29}$

27 Otto Ludwig, „Geschichte des Schreibens“, in: Hartmut Günther und Otto Ludwig (Hrsg.), Schrift und Schriftlichkeit. Writing and Its Use. Ein interdisziplinäres Handbuch internationaler Forschung, 1. Halbbd., Berlin, New York: de Gruyter 1994, S. 48-65, hier S. 59.

28 Auch hier wieder nur in bestimmten Ländern, Bildungsschichten oder Klassen. Vgl. Otto Ludwig, Der Schulaufsatz. Seine Geschichte in Deutschland, Berlin, New York: de Gruyter 1988, und Bosse, Bildungsrevolution (Anm. 1).

$29 \mathrm{Zu}$ einer solchen Soziologie Rainer Kuhlen, „Kollaboratives Schreiben“, in: Christoph Bieber und Claus Leggewie (Hrsg.), Interaktivität. Ein transdisziplinärer Schlüsselbegriff, Frankfurt/Main, New York: Campus 2004, S. 216-239. In der Linguistik und der Schreibdidaktik werden ,kollaborativ‘ und ,kooperativ‘ häufig gleich oder in Konkurrenz zueinander gebraucht. Besser ist, von ko-labor zu sprechen, um den Aspekt der Arbeit im Wort zu stärken. Das ermöglicht zugleich, unter Kooperativität die im Werk/Produkt (opus) vergegenständlichte, semantisierte, erzählte, dokumentierte Kollaboration zu verstehen, die im ,Werk' ausgeführte ,Herrschaft' also nicht auf einen Autor zu beziehen (oder beziehen zu müssen), sondern auf einen arbeitsteiligen Schreibprozess. Hier bietet sich eine Relektüre von Heinrich Bosse, Autorschaft ist Werkherrschaft. Über die 


\section{III.1}

Kommen wir also zu zwei Schlaglichtern aus der Literatur. Offenkundig spielt die Textsorte oder Gattung eine bedeutende Rolle für die Praxis des Schreibens, weil sie in jeweils andere, divergente Arbeitsformen eingebunden ist. Hervorragendes Beispiel ist die Theaterliteratur. David Lodge vermerkt in seinem Buch Handwerk des Schreibens, dass im Abstieg vom Roman zum Drama zum Fernsehspiel die individuelle Autorschaft graduell abnehme zu Gunsten oder - je nach Lesart - zu Ungunsten des gemeinsamen Schreibens am Text. ${ }^{30}$ Zur Logik des Theatertextes gehört, so könnte man sagen, diesen Umstand zu antizipieren oder zumindest im Vorhinein zu akzeptieren. Denn das Revidieren und Anpassen, insbesondere das Kürzen eines Textes für eine Aufführung sollte der Regel nach vorausgesetzt werden. Im Zuge der Überarbeitung hat man es dann mit einer Vielzahl an Beiträgen unterschiedlicher Hände zu tun, die insofern kollaborativ genannt werden können, als 1. einer oder mehrere hier einen zuvor von jemand anderem erstellten Text umarbeiten und den besonderen Anforderungen der Aufführung anpassen, und 2. die Umstände der Arbeitsteilung, die im Theaterbetrieb unmittelbar gegeben ist, diesen Vorgang mindestens indirekt erfordern, beeinflussen und bestimmen. ${ }^{31}$ Regie- und Textbücher sind dafür glänzende Beispiele, weil sie den Umstand der sich notwendig einstellenden Überarbeitung eines fertigen Textes zu einem Aufführungstext ihrer Bestimmung nach enthalten. In dem wunderbaren Buch Lesespuren - Spurenlesen. Wie kommt die Handschrift ins Buch? Von sprechenden und stummen Annotationen kann man sehen, wie das vor sich geht. Es konzentriert sich dabei auf die Annotation, also den Schreibanfang, mit dem eine literarische Arbeit geöffnet und umgeschrieben wird. ${ }^{32}$ So bezeugt das Textbuch und Arbeitsexemplar zu Grillparzers Sappho, wie viele

Entstehung des Urheberrechts aus dem Geist der Goethezeit, Paderborn u.a.: Schöningh 1981, an.

30 David Lodge, Das Handwerk des Schreibens (1996), aus dem Englischen übersetzt von Martin Ruf, Hamburg: Nikol 2007. „Tatsächlich kann man die drei Formen - Roman, Bühnenstück, Drehbuch - nach dem abnehmenden Grad der künstlerischen Kontrolle unterscheiden, die der Schriftsteller über die Form des Werks hat, in der es das Publikum rezipiert, und das wiederum ist mit den formalen Mitteln verbunden, die Autor und Publikum zur Kommunikation verwenden" (S. 1of.).

31 Welche Konflikte und welche Probleme dabei für den Begriff des Autors entstehen, hat Lodge in seiner semi-fiktiven Henry James-Biographie gezeigt: David Lodge, Autor, Autor (2004), aus dem Englischen übersetzt von Renate Orth-Guttmann, Frankfurt/Main: Haffmanns bei Zweitausendeins 2006.

32 Hrsg. im Auftrag des Literaturarchivs der Österreichischen Nationalbibliothek und der Wienbibliothek im Rathaus von Marcel Atze und Volker Kaukoreit, unter Mitarbeit von Thomas Degener, Tanja Gausterer und Martin Wedl, Wien: Praesens 2011. 
und welche Hände auf welche Weise an der Umarbeitung und Einrichtung des Stückes beteiligt sind: Dramaturgen, Regisseure, Schauspielerinnen, Souffleure, Inspizienten. Und je nach Interesse und Funktion fallen die Anmerkungen, Streichungen und Ergänzungen anders aus, heben sich gegenseitig auf, bestätigen sich oder nehmen Gestrichenes wieder zurück. ${ }^{33}$

Schreibszenen wie diese erhalten paradigmatischen Wert, wenn sich an ihnen die Frage entscheidet, wer im Prozess der Umarbeitung die Autorität des Autors behält bzw. wie das ökonomische zum ästhetischen Regime sich verhält. ${ }^{34}$ Das klassische Modell bietet Bertolt Brechts Dreigroschenoper bzw. Dreigroschenproze $\beta:{ }^{35}$ Die Dreigroschenoper zeigt beispielhaft das gemeinsame Arbeiten an einem Text, zu zweit, zu dritt usw., das Überarbeiten anderer Textewas Brecht bekanntlich einen Plagiatsvorwurf eingebracht hat-, die Aufteilung des Schreibens auf mehrere Köpfe und Hände, das Delegieren von Textabschnitten, und das alles mal simultan, parallel oder gestaffelt, schließlich mehr oder weniger zentralisiert auf einen verantwortlichen Autor, zudem multimedial weiter- und umgearbeitet, durch Musik, Aufführung, Schauspieler. ${ }^{36}$ Die Textebene ist durchzogen von den Händen der vielen Mitschreiberinnen und führt zu einer Reihe an Begleitereignissen und -texten, die unter anderen Schreibbedingungen gar nicht entstanden wären. Der Produktionsprozess wird poetisch aufgenommen und poetologisch reflektiert in den verschiedenen Beiträgen, die Brecht zum Theater und Kurt Weill zur Musik verfasst hat. Die besondere Konstellation unter den Schreibern und Schreiberinnen ist zugleich ein Beleg für strukturelle und patriarchale Hierarchien, die in der Verwertung und schließlich Monetarisierung des Stückes eine bedeutende Rolle spielen.

33 Walter Obermaier, „Erhabne, heil'ge Götter! / Ihr habt mit reichem Segen mich geschmückt!' Das Textbuch zu Franz Grillparzers Trauerspiel ,Sappho‘ aus dem Besitz von Charlotte Wolter", in: Atze/Kaukoreit, Lesespuren - Spurenlesen (Anm. 32), S. 211-216. Weitere Beispiele bieten in ebd. Friedrich Walla und Walter Obermaier, „Eine Posse in der nachrevolutionären Provinz. Ein Textbuch zu Nestroys ,Einen Jux will er sich machen“, S. 205-210; und Kurt Ifkovits, „,Schwer aufzuschreiben. Keine Noten fuer Sprechen'. Über Regiebücher im Allgemeinen und jene von Max Reinhardt im Besonderen“, S. 138-150.

34 Zur Begrifflichkeit: Friedrich Balke, Harun Maye und Leander Scholz (Hrsg.), Ästhetische Regime um 1800, München: Fink 2009.

35 Bertolt Brecht, Die Dreigroschenoper (1928), nach John Gays „The beggar's opera“, unter Mitarbeit von Elisabeth Hauptmann und Kurt Weill, Frankfurt/Main: Suhrkamp 1986; „Der Dreigroschenprozeß. Ein soziologisches Experiment“ (1931), in: ders., Gesammelte Werke, hrsg. vom Suhrkamp Verlag in Zusammenarbeit mit Elisabeth Hauptmann, Bd. VIII: Schriften 2. Zur Literatur und Kunst. Zur Politik und Gesellschaft, Frankfurt/Main: Suhrkamp 1967, S. 139-209.

36 Vgl. Walter Delabar, „Brechts Factory. Zur literarischen Produktion im Zeitalter der industriellen Arbeitsteilung“, in: Bodo Plachta (Hrsg.), Literarische Zusammenarbeit, Tübingen: Niemeyer 2001, S. 257-270. 
Theater in diesem Sinne radikalisiert die alte Maxime der Nachahmung von Handlung (Aristoteles) zur Nachahmung von Arbeit.

Im Dreigroschenproze $\beta$ wird diese Konstellation zu einer soziologischen Studie ausgearbeitet. Brecht verklagt die ausführende Filmkompanie, weil er sein Mitspracherecht an der Umarbeitung des Textes und der Inszenierung übergangen sieht. Im Prozess entwickelt sich die Frage, wer eigentlich der Besitzer der Oper und des Films ist. Jeder, der daran mitwirkt, die Besitzer der Rechte, der ursprüngliche Autor (und dessen Mitautorinnen)? Offenkundig wird, dass der Konflikt an den Grenzen der verschiedenen Diskurse verläuft (ästhetisch, juristisch, ökonomisch), die sich wiederum an der spezifischen Eigenart des Mediums beweisen müssen, das auf Kooperation und Kooperativität angelegt ist. Prüfstein der Auseinandersetzung ist also, inwieweit die Arbeitsprozesse im Film (und im Theater) auf die Besitz- und Auszahlungsverhältnisse der Beteiligten sich auswirken. Brecht besteht kurzerhand darauf, die Aufspaltung der künstlerischen Arbeit ökonomisch wie ästhetisch durchzusetzen, um auf diese Weise die Autonomie des Einzelnen und dessen Freiheit zu bewahren, nur eben unter anderen, fordistischen Bedingungen. ${ }^{37}$ Und betrachtet man nun - wenn auch an dieser Stelle reichlich knapp und eilig - die einzelnen Stationen dieser idealtypischen Theater- und Filmarbeit, von der Entstehung eines ersten Textes durch eine und mehrere Hände, zum Regiebuch, zur Inszenierung und Verfilmung, dann wird erstens deutlich, dass kollaboratives und kooperatives Schreiben stets im Verhältnis zur dominanten Arbeitsweise einer Gesellschaft betrachtet werden muss. Selbst dann, wenn es ,nur' um die kleinsten Schritte des Schreibens geht. Und zweitens, dass die diskursiven Zusammenstöße, die sich dabei ergeben (und sich auf Eigentumsverhältnisse und den Besitz an den Produktionsmitteln auswirken), auf die konkreten Arbeitsschritte zurück- und einwirken. Brechts Dreigroschenproze $\beta$ ist so gesehen Blaupause einer ganzen Reihe anderer Schreibformate (in der Serienproduktion z.B.), die das Schreiben über die Grenzen des Einzelnen hinaus zu ihrem Geschäftsmodell gemacht haben.

37 Zu Brechts ,Fordismus‘ am Beispiel des Dreigroschenprozesses Jan Knopf, Bertolt Brechts Erfolgsmarke. Dreigroschen für Fressen \& Moral, Stuttgart, Weimar: Metzler 2017, S. 21-25. Im Übrigen: Die Entdeckung des kollaborativen Schreibens in der Linguistik ist wesentlich Ergebnis medialer Innovationen, auch wenn dieser Aspekt bisher deutlich unterbelichtet ist, siehe etwa Almuth Grésillon und Daniel Perrin, „Methodology: From speaking about writing to tracking text production“, in: Jakobs/Perrin, Handbook of Writing (Anm. 21), S. 79-111, und Lilian Bridwell-Bowles, Parker Johnson und Steven Brehe, „Composing and Computers: Case Studies of Experienced Writers“, in: Ann Matsuhashi (Hrsg.), Writing in real time. Modelling Production Processes, Norwood/N.J.: Ablex 1987, S. 81-107. 


\section{III.2}

Heinrich Heine ist als Solitär und Ausnahmestilist kanonisiert worden, musste sich aber einer gesellschaftlichen Umgebung erwehren, die den Einzelnen verabschiedet, um an dessen Stelle die Masse und die Logik der kapitalistischen Arbeitsteilung zu setzen. Anders gesagt: In seiner Individualisierung des Stils stößt Heine auf die Macht des entindividualisierten Arbeitens. Ein Umstand, der im Pariser Exil an Bedeutung gewinnt, bis hin zur tragischen Situation in der ,Matratzengruft', die den schwer erkrankten Heine an Helfer, Schreiber und Sekretäre kettet. Was sich nun im Getriebe der Industrialisierung zunehmend durchsetzt, findet im Literaturbetrieb sein Gegenstück, ist es doch vor allem die Zeitung und die Vernetzung des Autors in einen Betriebsablauf, der neben Redakteuren und Verlegern, Druckern und Schreibern, Lesern, Kritikern und Gönnern auch den Zensor kennt, der in seiner Funktion als Diskursschranke die Bedingungen und Abhängigkeiten des Autors in nuce vorführt, Politik und Ästhetik in einer Person und Funktion verbindet. Im berühmten zwölften Kapitel des zweiten Teils der Reisebilder, Ideen. Das Buch le Grand, wird dieses komplexe Spiel paradigmatisch zugespitzt:

Die deutschen Zensoren

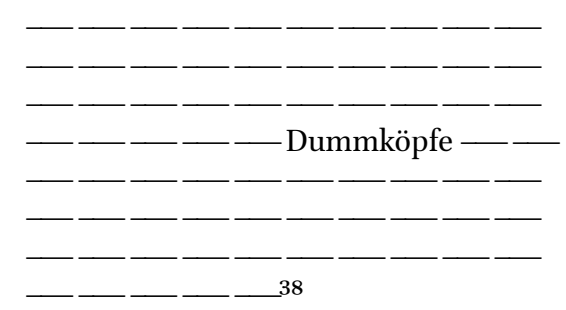

Es bietet eine gute Gelegenheit, es an zwei Schreibmodellen zu messen, einem linguistischen und einem literaturwissenschaftlichen.

John Hayes ist (zusammen mit Linda Flower) verantwortlich für eines der bekanntesten Schreibmodelle in der Linguistik und Schreibdidaktik. Vorteil des Modells ist generell, dass es die textgenetischen und generativpoetischen Aspekte des Schreibens prozessual darstellt, als eine Reihe von Praktiken und Kulturtechniken, die dem abgeschlossenen Text, dem Produkt, dem Werk vorausgehen. ${ }^{39}$ In der vorerst letzten Version aus dem Jahr 2012

38 Heinrich Heine, „Reisebilder. Zweyter Theil. Ideen. Das Buch Le Grand“ (1827), in: ders., Historisch-kritische Gesamtausgabe der Werke, Bd. 6, bearbeitet von Jost Hermand, Hamburg: Hoffmann und Campe 1973, S. 169-222, hier S. 201 („Capitel XII“).

39 Linda Flower und John Hayes, „A cognitive process theory of writing“, in: College Composition and Communication 32/4 (1981), S. 365-387. 


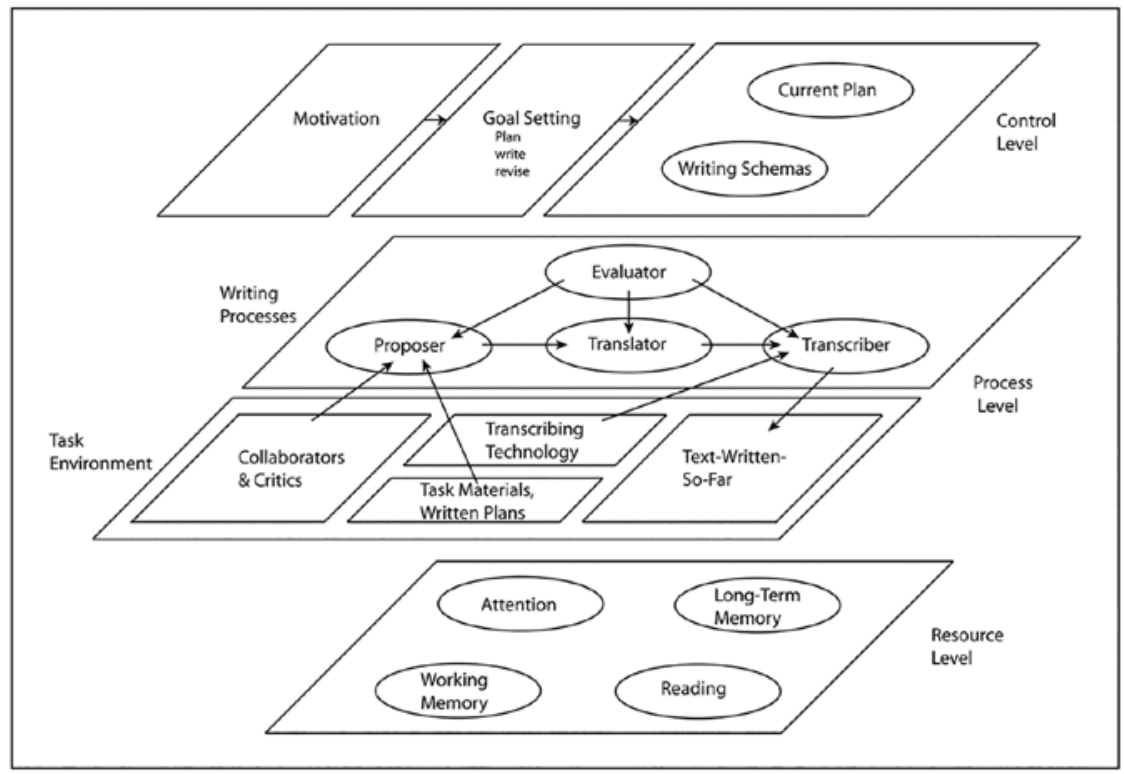

Abb. 13.1 John Hayes, Model of the writing process, 2012.

hat Hayes versucht, das kognitive Niveau des Schreibens auf drei Ebenen (Levels) wiederzugeben. ${ }^{40}$ Die Pfeilstruktur schafft dazu ein Netzwerk innerhalb der Ebenen, das Personen oder kognitive Fähigkeiten verbindet. Und mit etwas Phantasie kann man die verschiedenen Theorieansätze, die derzeit in den Geistes-, Kultur- oder Literaturwissenschaften in Verbindung mit dem ,Schreiben' auftreten, an dem Modell erproben und in Beziehung setzen. Von besonderem Interesse ist die mittlere Ebene. Was hier mit Collaborators \& Critics bezeichnet wird - die Station ist eine Innovation im Vergleich zu seinen Vorläufermodellen -, beeinflusst den gesamten Schreibprozess, und zwar nicht nur von Heines zwölftem Kapitel, sondern des ganzen Buches Le Grand, oder - wenn man so will - den einer ganzen Epoche (Vormärz, Nachmärz, Journalliteratur? - auch hier besteht Klärungsbedarf). Der Proposer, also wortwörtlich: der Antragsteller - Heine, das literarische Publikum, der Verleger, eine literarische Gruppe usf. - ist davon beeinflusst, der Evaluator, derjenige, der den Wert oder Schaden schätzt, genauso, und schließlich der Translator und der Transcriber, die all dies in Schriftsprache bringen müssen. Hayes'

40 John Hayes, „Modelling and Remodelling Writing“, in: Written Communication 29/3 (2012), S. $369-388$. 
Modell stellt dar, wahrscheinlich ungewollt, dafür aber auf der Ebene des Schreibprozesses, wo und auf welche Weise genau kollaborativ geschrieben wird. Im Schaubild fehlt nur noch ein Pfeil, zwischen Text-Written-So-Far und Collaborators \& Critics, um die Rückwirkungen der verschiedenen Stationen und Akteure auf den Schreibprozess in ein Kreislaufsystem sich gegenseitig beeinflussender Faktoren zu bringen.

Im Schreibszenenmodell, von Rüdiger Campe eingebracht, von Martin Stingelin erweitert und von einigen anderen modifiziert und weiterverarbeitet, liegt die Sache anders. ${ }^{41}$ Auch hier wird das Ereignis oder der Akt des Schreibens hervorgehoben, ohne allerdings auf kognitive Ressourcen (bei Hayes insbesondere Resource Level und Control Level) einzugehen. Stattdessen wird das Schreiben auf verschiedene Elemente hin ausgerichtet: Sprache, Instrumentalität, Materialität und Körperlichkeit/Geste. Deren Zusammenspiel in einer Szene stellt die Frage nach Regie und Rolle der im Schreibakt beteiligten Akteure, wobei darunter nicht nur der Autor zu verstehen ist, sondern auch die beteiligten Schreibinstrumente, Beschreibstoffe, körperlichen Zustände usf. Die politischen und historischen Bedingungen des Schreibens schließlich werden als ,Begleitumstände' geführt (im zitierten Modell mit ,Leben` und ,Diskurs` wiedergegeben). ${ }^{42}$,Poetisch` ist die SchreibSzene dann, wenn das Schreiben sich selbst zum Thema wird und bestenfalls, in der dokumentarischen Schreibszene, Spuren und Zeugnisse des Schreibprozesses hinterlässt - eine (medien-)philologische Dimension, die in Hayes' Modell fehlt.

Anders als bei Hayes, Schindler und Wolfe führt bereits die Materialität und Instrumentalität des Schreibens zu ,Kollaborationen', noch vor der - wie in den Modellen der Linguistik - personal gedachten Akteursebene. Schließlich können die verschiedenen Elemente selbst Gegenstand des Schreibens werden, explizit oder implizit. Das ist für das gegebene Beispiel besonders interessant, handelt es sich hier doch um eine inszenierte Zensur, die sehr deutlich die verschiedenen Umstände des kollaborativen Schreibens - im Guten wie im Schlechten - vor Augen führt. Der zensierte Text setzt einen unzensierten, autonomen Text voraus, der in den Auslassungen erkennbar wird. Wer die Produktionsbedingungen im literarischen Feld in der ersten Hälfte des

41 Zusammenfassend Martin Stingelin, „Schreiben“, in: Jan-Dirk Müller et al. (Hrsg.), Reallexikon der deutschen Literaturwissenschaft, Bd. III, Berlin, New York: de Gruyter 2003, S. $387-389$.

42 Vgl. auch Hubert Thüring, „Konstruktion und Dekonstruktion der biopolitischen Schreibszene“, in: Verf., Matthias Thiele und Martin Stingelin (Hrsg.), Die Schreibszene als politische Szene, München: Fink 2012, S. 109-128, hier S. 119. 


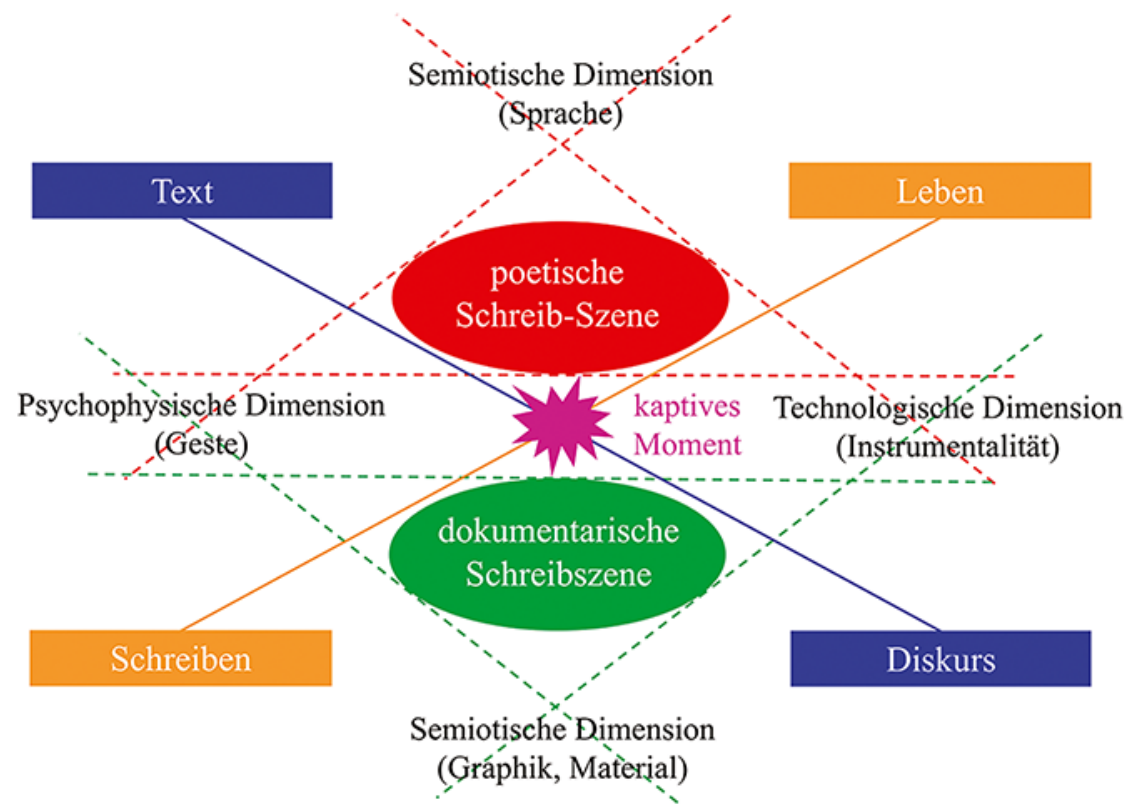

Abb. 13.2 Hubert Thüring, Modell der Schreibszene, 2012.

19. Jahrhunderts mit einbezieht, der weiß, dass damit auch eine medientechnische Zäsur einhergeht, nämlich die zwischen Manuskript und Druck. Mit der Drucklegung wird aus dem individuellen Schreiben ein kollaboratives und kooperatives, das auf verschiedene Instanzen angewiesen ist, die am Text mitschreiben, im Kleinen - beim Setzen der Druckvorlage - wie im Großen in den Auseinandersetzungen mit dem Verleger und/oder den politischen und ökonomischen Umständen. Nun wirken die technischen und politischen Umstände wieder zurück auf den Schreibprozess der Manuskriptebene, der bereits antizipiert, dass der Text einmal gedruckt werden soll. Insofern verdeutlicht das Beispiel Heines nicht nur, wie man klug mit der Zensur umgehen kann, sondern auch, dass die Zensur nicht erst am Ende des Schreibens, im Zuge der Drucklegung eine Rolle spielt, sondern selbstverständlich in allen Stadien und auf allen Ebenen des Schreibprozesses. Das betrifft das Planen, Entwerfen, Beauftragen, Entwickeln, Überarbeiten, Revidieren so gut wie die Textstruktur im textlinguistischen Sinne, also Aspekte der Kohärenz und Kohäsion.

In diesem Fall hat Heine gemeinsam mit den deutschen Zensoren einen Zusammenhang hergestellt, der - womöglich - zuvor gar nicht da war, und zwar indem er einen elliptischen Satz baut, der - weil er unvollständig ist -, nur 
umso vollständiger die politischen Umstände des Schreibens zum Ausdruck bringt. Oder anders: Im ,kaptiven Moment' stoßen Diskurs und Text, poetische und dokumentarische Schreibszene, Leben und Schreiben zusammen. Heines Text ist selbstredend eine Provokation, die aber - wenn man sie methodologisch zu Ende denkt - Konsequenzen für jedes Schreibmodell hat, das die Bedingungen des Schreibens im Zeichen einer arbeitsteiligen, politisch und ökonomisch durchsetzten Gesellschaft mit einbeziehen möchte. Man kann sich das an der vielzitierten ,Schere im Kopf' vorstellen. Die Collaborators \& Critics, einschließlich der Zensoren, müssen nicht reale Personen oder Akteure einer Institution sein, sie lassen sich auch als Funktionen beschreiben, die in einer Person oder im Schreibprozess Konflikte erzeugen, die wie im Theater szenisch zur Aufführung bringen, was politisch genehm ist und was nicht. Bei Niklas Luhmann ist zu lesen: „Nur die Kommunikation selbst ist sozial. Schreiben und Lesen muß man zwangsläufig allein, und wenn andere dabei sind und zuschauen, ist eine zu intensive Beobachtung nutzlos, indiskret und verdächtig“. ${ }^{43}$ Schreiben ist so gesehen nicht nur kollaborativ und kooperativ, weil es die Mitarbeit vieler Hände braucht, ob sie nun erwünscht sind oder nicht, in der Vorstellung ihr Regiment ausüben oder ganz konkret. Es ist auch verdächtig. ${ }^{44}$

43 Niklas Luhmann, Die Gesellschaft der Gesellschaft, Frankfurt/Main: Suhrkamp 1998, S. 274 f.

44 Unter welchen Bedingungen es dann auch noch kollektiv sein kann, steht auf einem anderen Blatt. Vgl. Verf., „Kollaboratives Arbeiten und kollektives Schreiben“, in: Undercurrents. Forum für linke Literaturwissenschaft 7 , https://undercurrentsforum.com/ indexphp/undercurrents/article/view/5o (letzter Zugriff: 28.02.2019). 